\title{
ARTICLE
}

\section{A convenient and cost efficient route suitable for "one-pot" synthesis of molnupiravir}

Received 00th January 20xx, Accepted 00th January 20xx DOI: $10.1039 / x 0 x \times 00000 x$
Tianwen Hu, ${ }^{\text {\&ac }}$ Yuanchao Xie, ${ }^{\text {\&b }}$ Yin Liu, ${ }^{\mathrm{bc}}$ Haitao Xue, ${ }^{\mathrm{bc}}$ Fuqiang Zhu, ${ }^{\mathrm{d}}$ Haji A. Aisa, ${ }^{* a c}$ and Jingshan Shen ${ }^{*}$ bc

\begin{abstract}
A convenient and cost efficient route to molnupiravir from cytidine has been developed comprising protection-assisted acylation and transamination. The site-specific esterification is easily achieved by using $\mathrm{N}, \mathrm{N}$-dimethylformamide dimethyl acetal (DMF-DMA) as an extraordinary protecting agent, and provides the key 5 '-isobutyrate intermediate in $92 \%$ yield. Subsequent hydroxyamination gives the product in $92 \%$ yield. Notably, this synthesis is very suitable for "one-pot" process, which affords this crucial drug candidate (purity up to $99 \%, \mathrm{HPLC}$ a/a) in $70 \%$ yield without chromatography purification. The new approach is superior to the existing methods in aspect of the yield, the cost and the operation, and shows great potential value for industrial application. Additionally, DMF-DMA is also successfully used in the synthesis of remdesivir from its parent nucleoside and the overall yield is much higher than that of the synthesis using acetonide protection.
\end{abstract}

\section{Introduction}

The COVID-19 pandemic caused by the SARS-CoV-2 virus has posed a great threat to public health. Globally, by the end of March $8^{\text {th }}, 2021$, more than 115 million people have been infected and nearly 2.5 million people were dead ${ }^{1}$. Therefore, there is an urgent need to discover effective antivirals to tackle the health crisis ${ }^{2}$. Molnupiravir (also known as EIDD-2801, MK4482) (1 in Fig.1), an orally available ribonucleoside analogue,

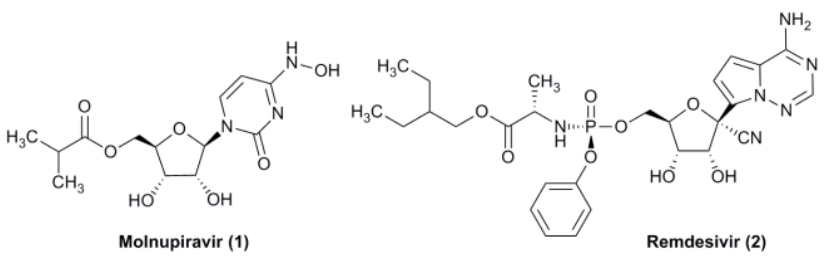

Figure 1. The chemical structures of molnupiravir and remdesivir

being evaluated in Phase $\mathrm{I} / \mathrm{III}$ clinical trials, is a promising experimental drug for the treatment of COVID-19 ${ }^{3}$. Similar to but not the same as remdesivir (RDV) ( 2 in Fig.1) which is an

\footnotetext{
State Key Laboratory Basis of Xinjiang Indigenous Medicinal Plants Resource Utilization, Xinjiang Technical Institute of Physics and Chemistry, Chinese Academy of Sciences, South Beijing Road 40-1, Urumqi, 830011, P.R. China.E-mail: haji@ms.xjb.ac.cn

b. Shanghai Institute of Materia Medica, Chinese Academy of Sciences, 555 Zuchongzhi Road, Shanghai 201203, P.R. China. E-mail: shenjingshan@simm.ac.cn.

c. University of Chinese Academy of Sciences, No. 19A Yuquan Road, Beijing 100049, P. R. China

${ }^{d .}$ Topharman Shanghai Co., Ltd., No.388 Jialilue Road, Zhangjiang Hitech Park, Shanghai 201203 P.R. China

†.Electronic Supplementary Information (ESI) available: See DOI: $10.1039 / x 0 x x 00000 x$

${ }^{\&}$ These authors contributed equally
}

intravenous nucleotide prodrug against COVID-19 approved by $\mathrm{FDA}^{4}$, this antiviral candidate exerts anti-SARS-CoV-2 effect by lethal mutagenesis rather than chain termination ${ }^{5-7}$, and may demonstrate synergic anti-SARS-CoV-2 effect with remdesivir ${ }^{8}$.

With respect to the synthesis of molnupiravir, the initially reported route from uridine consisted of five steps (acetonide protection of 2',3' hydroxyl groups, esterification, triazole coupling, hydroxyamination and deprotection) ${ }^{9}$ (Scheme 1a).

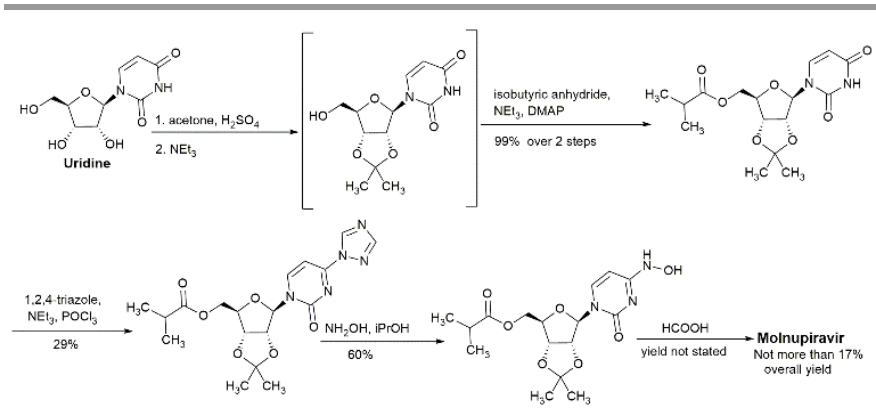

Scheme 1a. The initial route to molnupiravir from uridine

These reactions were simple, but the overall yield was not more than $17 \%$. The low overall yield was mainly attributed to the poor conversion rate in the triazloe coupling step. Recently, Kappe and co-workers reported an improved route reordering the reaction sequence ${ }^{10}$ (Scheme $1 b$ ). Meanwhile, the continuous flow technology was used, which minimized side product production in the final acetonide deprotection step. Although the overall yield was improved to $61 \%$, two impurities $A$ and $B$ resulting from deprotection still remained in the reaction mixture, which posed a challenge to the separation and purification of the product. 

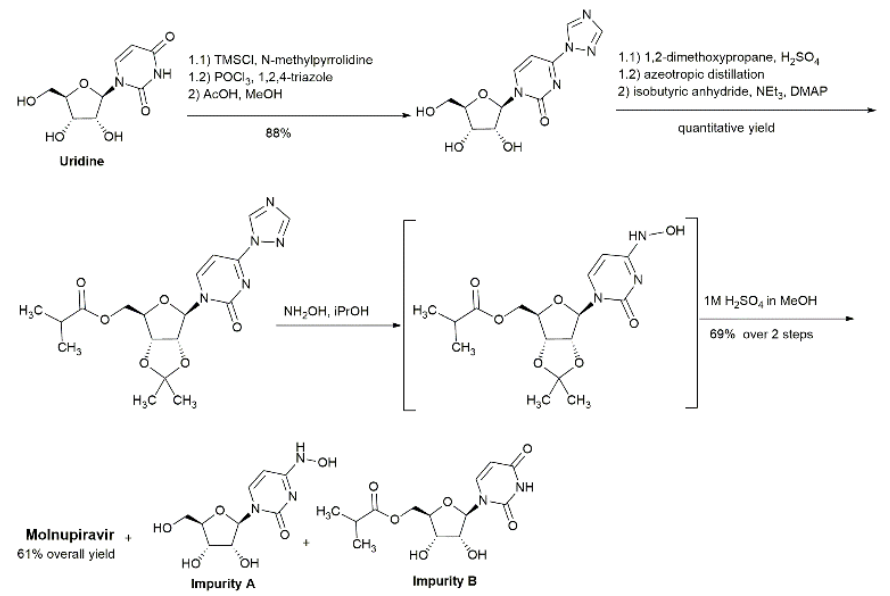

Scheme $1 \mathrm{~b}$. The improved route to molnupiravir from uridine and impurities in deprotection

Nearly at the same time, Snead and co-workers reported a two-step concise synthesis of molnupiravir in a total yield of $75 \%{ }^{11}$ by column chromatography (Scheme $1 \mathrm{c}$ ). This route was characterized by enzyme-mediated selective esterification and direct hydroxyamination, but the cost of this method limited its industrial application. In addition, they provided an enzymefree synthetic route from cytidine, which was similar with the initially reported route except for the hydroxyamination step $^{12}$ (Scheme 1d). This synthesis provided a relative higher overall yield (44\%) than that of the initial synthesis, but it also suffered from the hydrolyzed impurities in the final step due to the use of acetonide protection.

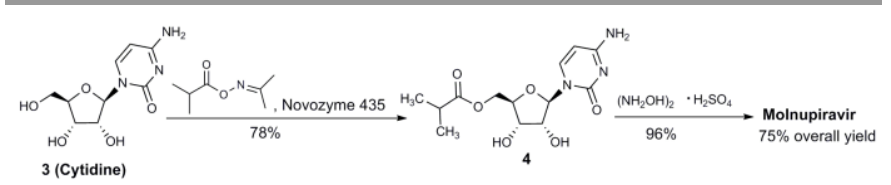

Scheme 1c. The enzyme-mediated route to molnupiravir from cytidine
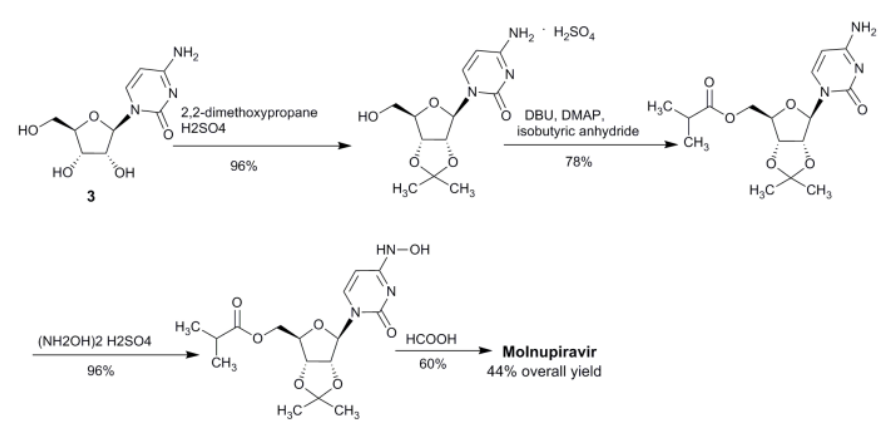

Scheme $1 \mathrm{~d}$. The enzyme-free route to molnupiravir from cytidine

Though some improvements have been achieved in the synthesis of molnupiravir, it is still worthy to develop a more facile and efficient method which is highly acceptable for industrial application. It is evident that the key to the achievement of the goal is to find an appropriate protecting group masking the 2', 3'-dihydroxyl groups as well as being deprotected readily under mild conditions. Herein, we discovered that $\mathrm{N}, \mathrm{N}$-Dimethylformamide dimethyl acetal (DMF-DMA) could serve as an excellent protecting agent, which greatly improved the preparation of this investigating drug from commercially available cytidine. Based on that, a convenient and cost efficient one-pot synthesis of molnupiravir was further carried out, which would facilitate a large-scale production of this antiviral candidate. Moreover, we also successfully used this protecting agent to accomplish the synthesis of remdesivir in an acceptable yield.

\section{Result and discussion}

In previous study ${ }^{13}$, DMF-DMA was used to provide the 2', 3'$O$-dimethylaminomethylene protected nucleosides which was used to achieve the tritylation at the $5^{\prime}$-hydroxyl group of nucleosides in spite of the inherent instability of 2', 3'-Odimethylaminomethylene group. Inspired by this work, we tried to synthesize the key ester $\mathbf{5}$ using this agent by three successive reactions, condensation with DMF-DMA, esterification with isobutyric anhydride and deprotection (Scheme 2). Both intermediates 5-1 and 5-2 were prone to degrade on the TLC, hence the reaction process could be determined by the degree of material consumption. Firstly, treatment cytidine with DMF-DMA in the pyridine at ambient

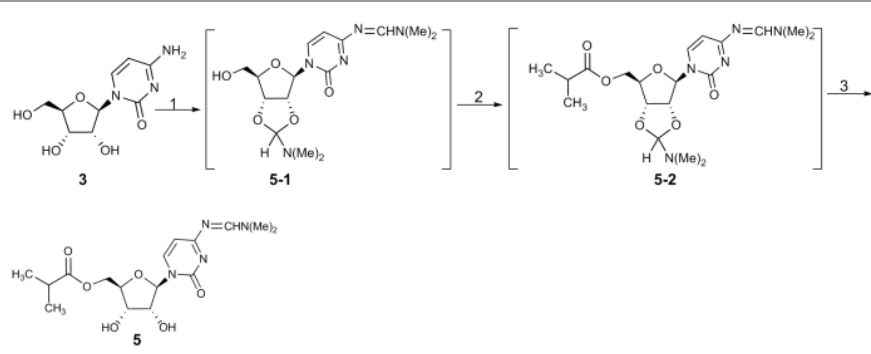

Scheme 2. The preparation of $\mathbf{5}$ form $\mathbf{3}^{\mathrm{a}}$. ${ }^{\mathrm{a}}$ Reaction conditions: 1) DMF-DMA, pyridine, $\mathrm{rt}$ overnight; 2 ) isobutyric anhydride, NEt3, DMAP, dichloromethane, rt, $2 \mathrm{~h} ; \mathrm{c}$ ) ethanol, dichloromethane, $\mathrm{rt}, 2 \mathrm{~h}$.

temperature overnight gave $\mathbf{5 - 1}$ in quantitative yield. Since pyridine was an unwelcome solvent from an environmental point. The reaction conditions, especially the solvent, were screened, and the result was shown in Table 1. It was observed that the reaction conversions were below $20 \%$ for all the three solvents (dichloromethane, toluene and THF) in the absence of pyridine. When 10.0 equivalent of pyridine was used, the conversions were improved, and THF gave the best result at refluxing temperature with more than $95 \%$ conversion. Due to methanol released from the reaction, the operation of concentration was required to avoid the influence of this byproduct on the subsequent esterification. After solvent evaporation, the resulting crude 5-1 was dissolved in dichloromethane, followed by the sequential addition of triethylamine, $N, N$-dimethylaminopyridine (DMAP) and isobutyric anhydride. The esterification went smoothly to give 5-2 at ambient temperature. Subsequently, ethanol was added to quench the reaction and concomitant deprotection of 2', 3'$O$-dimethylaminomethylene group happened, affording the critical intermediate 5 with an excellent yield (92\%) through column chromatography. Furthermore, we found that $\mathbf{5}$ was easily crystallized by treatment of the crude product with a 
mixture of isopropyl acetate (iPrOAc) and methyl tert-butyl ether (MTBE) in ratio of $\sim 2: 1$.

Table 1. Optimization of the reaction of cytidine with DMF-DMA ${ }^{a}$

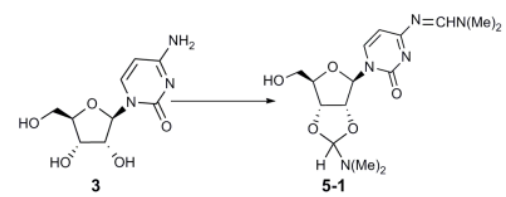

\begin{tabular}{|c|c|c|c|c|}
\hline Entry & Solvent & Base (eqiv.) & $T\left[{ }^{\circ} \mathrm{C}\right]$ & Conv. [\%] \\
\hline 1 & Pyridine & - & $\mathrm{rt}$ & $>95$ \\
\hline 2 & Dichloromethane & - & $\mathrm{rt}$ & $<20$ \\
\hline 3 & Toluene & - & $\mathrm{rt}$ & $<20$ \\
\hline 4 & THF & - & $\mathrm{rt}$ & $<20$ \\
\hline 5 & Dichloromethane & Pyridine (10eq) & $\mathrm{rt}$ & $<50$ \\
\hline 6 & Toluene & Pyridine (10eq) & $\mathrm{rt}$ & $<50$ \\
\hline 7 & THF & Pyridine (10eq) & $\mathrm{rt}$ & $<50$ \\
\hline 8 & Dichloromethane & Pyridine (10eq) & 40 & $\sim 80$ \\
\hline 9 & Toluene & Pyridine (10eq) & 80 & $\sim 80$ \\
\hline 10 & THF & Pyridine (10eq) & reflux & $>95$ \\
\hline
\end{tabular}

${ }^{\mathrm{a}}$ Reaction conditions: cytidine $(486 \mathrm{mg}, 2.0 \mathrm{mmol}$, 1eq), DMF-DMA (952mg, $8.0 \mathrm{mmol}, 4 \mathrm{eq})$; $^{\mathrm{b}}$ Determined by TLC analysis

The exocyclic $N$-protection of $\mathbf{5}$ was removed using 20.0 equiv of acetic acid in ethanol to give 4 with a yield of $90 \%$ after chromatography. Referring to the reported method ${ }^{11}$, treatment 4 with hydroxylamine sulfate afforded 1 in $90 \%$ yield with chromatography purification (Scheme 3 ). At this time, we successfully synthesized 1 from cytidine with DMFDMA as a protecting agent, and this new method only consisted of three steps with an overall yield up to $75 \%$, which was more economic than the known enzyme-catalyzed method $^{11}$.

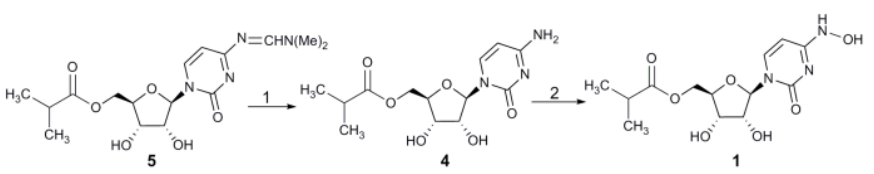

Scheme 3. The preparation of $\mathbf{1}$ from $\mathbf{5}$ through two steps ${ }^{\mathrm{a}}$. ${ }^{\mathrm{a}}$ Reaction conditions: 1) acetic acid, ethanol, rt, $2 \mathrm{~h}$; b) hydroxylamine sulfate, $70 \% \mathrm{iPrOH}$ aqueous solution, $78^{\circ} \mathrm{C}, 18 \mathrm{~h}$

Next, we attempted to investigate the direct transformation from $\mathbf{5}$ to $\mathbf{1}$, which was based on that the amino group of cytidine could be replaced by hydroxylamine in a high yield. With this idea in mind, the reaction was performed under similar conditions. Reaction of $\mathbf{5}$ with hydroxylamine sulfate in isopropanol (iPrOH) at $78^{\circ} \mathrm{C}$ successfully afforded the target product with a yield up to $92 \%$ by chromatography purification. The result was exciting and far beyond our expectation. In the course of the reaction, two intermediates (1-1 and 4) were identified, which indicated the conversion from the exocyclic amidine analogue $\mathbf{5}$ to the hydroxyamination product $\mathbf{1}$ went through a multi-step process (Scheme 4). Obviously, 1-1 was derived from the substitution at the dimethylamino group of $\mathbf{5}$ by hydroxylamine. When the reaction was conducted at room temperature in $70 \%$ iPrOH aqueous solution, 1-1 was formed rapidly, and remained unchanged under this condition. After raising the reaction temperature to $78^{\circ} \mathrm{C}$, it was observed that intermediate 1-1 was gradually converted to 4 , and as the reaction time went on, both 1-1 and 4 were disappeared, giving $\mathbf{1}$ as the predominant product. Therefore, the mechanism of the conversion from $\mathbf{5}$ to $\mathbf{1}$ was figured out, which was a three-step reaction process.

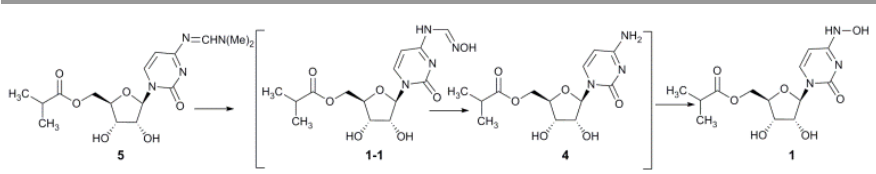

Scheme 4. The preparation of $\mathbf{1}$ from $\mathbf{5}$ through one step ${ }^{\mathrm{a}}$. ${ }^{\mathrm{a}}$ Reaction condition hydroxylamine sulfate, $70 \% \mathrm{iPrOH}$ aqueous solution, $78^{\circ} \mathrm{C}, 18 \mathrm{~h}$

Given the hydroxyamination mechanism of $\mathbf{5}$, the residual triethylamine and DMAP in the esterification would have no effect on the final reaction. At the same time, both the esterification and the hydroxyamination were performed in excellent yield. These facts urged us to prepare $\mathbf{1}$ from cytidine in one-pot method. Gratifyingly, this method was achieved successfully with $70 \%$ overall yield by crystallization (Scheme 5). The process of this method was as follow: Cytidine was dried by co-evaporation with tetrahydrofuran, and reacted with DMF-DMA (4.0eq) in the presence of pyridine (10.0eq) in THF subsequently. After stirring overnight, the reaction mixture was concentrated to give the crude 5-1. Treatment of

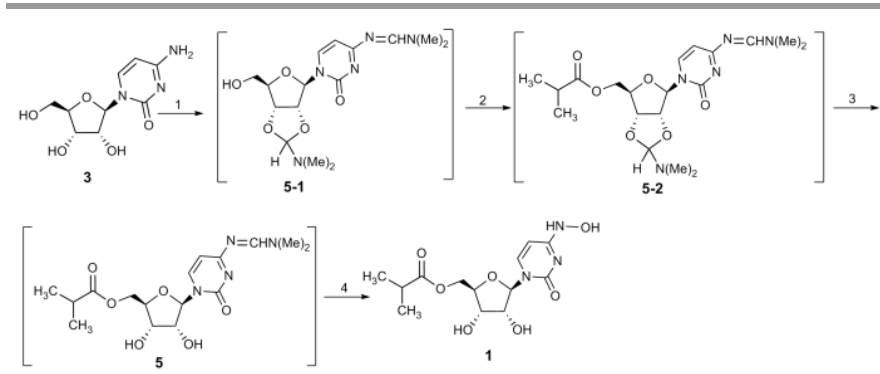

Scheme 5. The preparation of 1 from $\mathbf{3}$ by "one-pot"method ${ }^{\mathrm{a}}$. 'Reaction conditions: a) DMF-DMA, pyridine, rt, overnight; b) isobutyric anhydride, NEt3, DMAP, dichloromethane, $\mathrm{rt}, 2 \mathrm{~h}$; $\mathrm{c}$ ) ethanol, dichloromethane, $\mathrm{rt}, 2 \mathrm{~h}$; d)hydroxylamine sulfate, $70 \% \mathrm{iPrOH}$ aqueous solution, $78^{\circ} \mathrm{C}, 18 \mathrm{~h}$.

this protected cytidine with isobutyric anhydride (1.5eq) in the presence of triethylamine (2.0eq) and DMAP (0.05eq) in dichloromethane at room temperature for $2 \mathrm{~h}$ afforded 5-2. When the conversion was finished, ethanol was added into the reaction mixture and the deprotection of 2', 3'-Odimethylaminomethylene group was occurred, providing $\mathbf{5}$ simultaneously. The crude $\mathbf{5}$ was evaporated with iPrOH. Subsequent hydroxamination by hydroxylamine sulfate ( $4.0 \mathrm{eq})$ at $78^{\circ} \mathrm{C}$ for $18 \mathrm{~h}$ gave the product 1 . When the whole process was completed, the reaction mixture was cooled to the ambient temperature and separated into two layers. The solvent of the organic layer was removed under reduced pressure and the resulting mixture was dissolved in 2-Methyl THF. The aqueous layer was diluted with water, which was used to wash the 2-Methyl THF solution. After concentration 
of the washed 2-Methyl THF solution, the crude product was obtained and reslurried in iPrOAc to afford the pure 1 (purity up to $99 \%, \mathrm{HPLC}$ a/a) as white solid. On the whole, the one-pot method involving DMF-DMA afforded high pure molnupiravir in an excellent yield of $70 \%$ with simple operations and being free of chromatography purification, which was preferable to previous methods, and had great potential value for commercial production.

Similar to the synthesis of molnupiravir, a protecting agent was also employed to mask 2', 3'-dihydroxyl groups in the preparation of RDV (2). As shown in Scheme $6^{14}, 2$ was prepared from its parent nucleoside $\mathbf{6}$ through three transformations, acetoinde protection, phosphorylation and deprotection. The total yield over three steps was about $43 \%$, which was partially attributed to the hydrolysis of the ester in the prodrug moiety during the final deprotection. Consequently, a better protection could be conducive to the improvement of the overall yield.
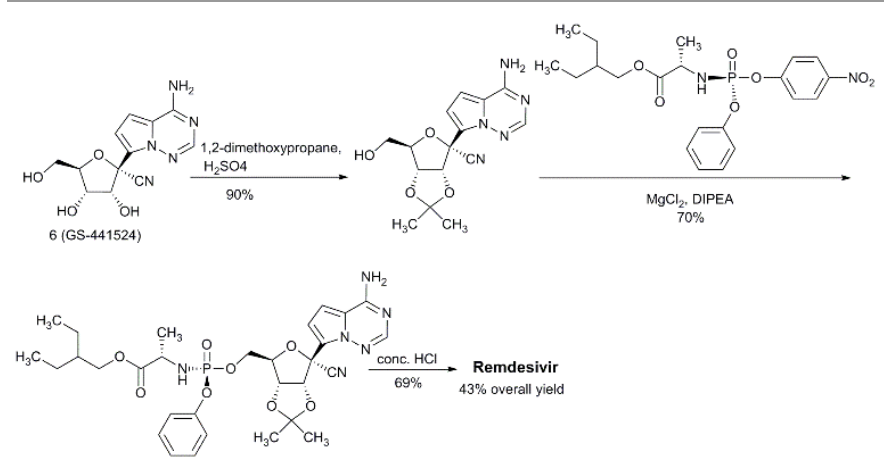

Scheme 6 . The preparation of remdesivir from 6 using acetoinde protection

Encouraged by the successful application of DMF-DMA in the synthesis of molnupiravir, we envisioned that this protecting agent may favor the synthesis of the prodrug 2. Along this line, we designed a two-step synthetic route with 6 as the starting material (Scheme 7). The first step contained

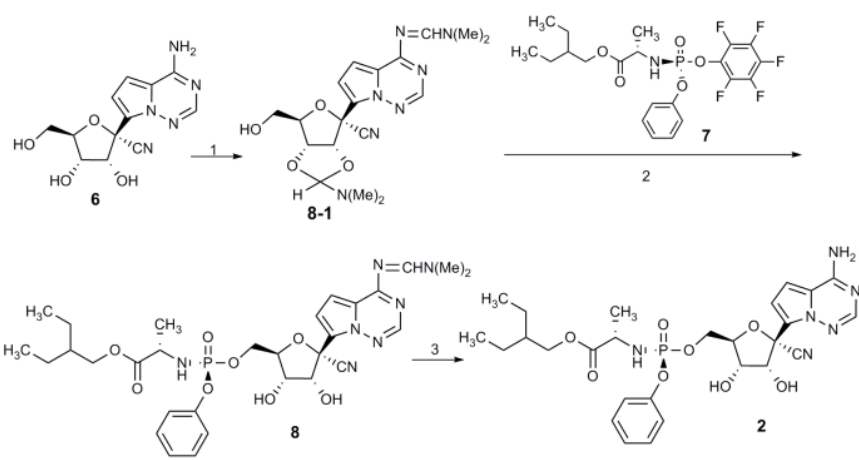

Scheme 7. The preparation of $\mathbf{2}$ from 6 using DMF-DMA as a protecting agent ${ }^{\mathrm{a}}$. ${ }^{\mathrm{a}}$ Reaction conditions: 1). DMF-DMA, pyridine, rt, overnight; 2). (i) $\mathrm{CH}_{3} \mathrm{MgBr}, \mathrm{THF},-15^{\circ} \mathrm{C}$ to $0^{\circ} \mathrm{C}, 3 \mathrm{~h}$, (ii) saturated $\mathrm{NH}_{4} \mathrm{Cl}$ solution, $\mathrm{THF}, \mathrm{rt}, 1 \mathrm{~h} ; 3$ ).acetic acid, ethanol, $50^{\circ} \mathrm{C}$ overnight

three successive reactions. Reaction of 6 with DMF-DMA in pyridine gave the crude $\mathbf{8 - 1}$, which was subsequently coupled with the optically pure phosphorylating agent $\mathbf{7}$ in THF with methyl magnesium bromide as the deprotonating agent. When the reaction was finished, $\mathrm{NH}_{4} \mathrm{Cl}$ aqueous solution was added to the reaction mixture, and 2',3'-O-dimethylaminomethylene group was removed concomitantly to give the exocyclic $N$ protection analogues $\mathbf{8}$ in $85 \%$ yield after chromatography purification. The following deprotection was conducted with acetic acid in ethanol to give the product $\mathbf{2}$ in $90 \%$ yield by chromatography. Generally, the new method with DMF-DMA as the hydroxyl protecting group provided an improved yield compared with the existing method.

\section{Conclusions}

In summary, we have developed an easy method to synthesize molnupiravir and remdesivir utilizing DMF-DMA as a protecting agent. For molnupiravir, a convenient and cost efficient one-pot synthesis is described from cytidine, and the high pure product (purity up to $99 \%$, HPLC a/a) is obtained in $70 \%$ overall yield without chromatography purification. This method offers several significant advantages in terms of the yield, the cost and the operation, and has huge potential for industrial application. For remdesivir, the overall yield of the new synthetic route from its parent nucleoside 6 is improved to $76 \%$.

\section{Conflicts of interest}

There are no conflicts to declare.

\section{Acknowledgements}

This work was supported financially by the National Science \& Technology Major Project "Key New Drug Creation and Manufacturing Program", China (Number: 018ZX09711002) and 2020 ANSO Collaborative Research Project (number: ANSO-CR-SP-2020-03).

\section{Notes and references}

1 N.D.Grubaugh,E.B.Hodcroft,J.R.Fauver,A.L.PhelanandM.Cevik, Cell,2021,DOI:10.1016/j.cell.2021.01.044.

2 J. T. Jan, T. R. Cheng, Y. P. Juang, H. H. Ma, Y. T. Wu, W. B. Yang, C. W. Cheng, X. Chen, T. H. Chou, J. J. Shie, W. C. Cheng, R. J. Chein, S. S. Mao, P. H. Liang, C. Ma, S. C. Hung and C. H. Wong, Proc Natl AcadSci U S A, 2021, 118.

3 https://www.cortellis.com/drugdiscovery/entity/drug/1066 867/product?ent $=5$ zvExtD0.

4 J. J. Malin, I. Suárez, V. Priesner, G. Fätkenheuer and J. Rybniker, Clin Microbiol Rev,2020, 34, e00162-00120.

5A. J. Pruijssers and M. R. Denison, CurrOpinVirol, 2019, 35, 5762.

6 N. R. Jena, Phys Chem Chem Phys, 2020, 22, 28115-28122.

7 W. Yin, C. Mao, X. Luan, D. D. Shen, Q. Shen, H. Su, X. Wang, F. Zhou, W. Zhao, M. Gao, S. Chang, Y. C. Xie, G. Tian, H. W. Jiang, S. C. Tao, J. Shen, Y. Jiang, H. Jiang, Y. Xu, S. Zhang, Y. Zhang and H. E. Xu, Science (New York, N.Y.), 2020, 368, 1499-1504.

8 https://trialsitenews.com/merck-positioning-to-take-the-leadu-s-covid-19-antiviral-market-from-remdesivir-eidd-2801/

9 G. R. Painter, D. Perryman and G. R. Bluemling, WO2019173602, 2019. 
10 A. Steiner, D. Znidar, S. B. Ötvös, D. R. Snead, D. Dallinger and C. O. Kappe, European Journal of Organic Chemistry, 2020, 2020, 6736-6739.

11 N. Vasudevan, G. P. Ahlqvist, C. P. McGeough, D. J. Paymode, F. S. P. Cardoso, T. Lucas, J. P. Dietz, T. Opatz, T. F. Jamison, F. B. Gupton and D. R. Snead, Chem Commun (Camb), 2020, 56, 13363-13364.

12 D. R. Snead, V. Gopalsamuthiram, C. Williams, J. Noble, T. F. Jamison and B. F. Gupton, Synlett, 2020, 32, 326-328.

13 Q. Song, W. Wang, A. Fischer, X. Zhang, B. L. Gaffney and R. A. Jones, Tetrahedron Letters, 1999, 40, 4153-4156.

14 T. K. Warren, R. Jordan, M. K. Lo, A. S. Ray, R. L. Mackman, V. Soloveva, D. Siegel, M. Perron, R. Bannister, H. C. Hui, N. Larson, R. Strickley, J. Wells, K. S. Stuthman, S. A. Van Tongeren, N. L. Garza, G. Donnelly, A. C. Shurtleff, C. J. Retterer, D. Gharaibeh, R. Zamani, T. Kenny, B. P. Eaton, E. Grimes, L. S. Welch, L.Gomba, C. L. Wilhelmsen, D. K. Nichols, J. E. Nuss, E. R. Nagle, J. R. Kugelman, G.Palacios, E. Doerffler, S. Neville, E. Carra, M. O. Clarke, L. Zhang, W. Lew, B. Ross, Q. Wang, K. Chun, L. Wolfe, D. Babusis, Y. Park, K. M. Stray, I. Trancheva, J. Y.Feng, O. Barauskas, Y. Xu, P. Wong, M. R. Braun, M. Flint, L. K. McMullan, S. S. Chen, R. Fearns, S. Swaminathan, D. L. Mayers, C. F. Spiropoulou, W. A. Lee, S. T. Nichol, T. Cihlar and S. Bavari, Nature, 2016, 531, 381385. 